\title{
Object Detection In Image Processing Using Edge Detection Techniques
}

\author{
A.Ushma, M.phil Scholar, ${ }^{1}$ Prof. Dr. A.R. Mohamed Shanavas, \\ M.Sc., P.G.D.C.A., M.Ed., M.phil, Ph.D., ${ }^{2}$ \\ I'Department of Computer Science, Jamal Mohamed College, Tirchy, India.) \\ ${ }^{2}$ (Associate Professor, Department of Computer Science, Jamal Mohamed College, Tirchy, India.)
}

\begin{abstract}
Object detection is the task of finding and identifying objects in an image. Humans recognize a multitude of objects in images with little effort, despite the fact that the image of the objects may vary somewhat in different viewpoints and sizes. This paper presents a survey on methods that use digital image processing techniques to detect the object from digital images. Basically to detect an object here we use two major steps: image enhancement and edge detection. To detect a object from digital images is major task in image processing. It is a hot issue all over the world to detect and classify the shape based on image processing technology.
\end{abstract}

Keywords: - Shape detection, image enhancement, edge detection.

\section{INTRODUCTION}

"One picture is worth more than thousand words." A digital image is a representation of two dimensional image as a finite set of digital values, called picture elements or pixels.

There are different types of images available. Black and white, Gray scale and color images. Image enhancement is the process of improving the quality of a digitally stored image.

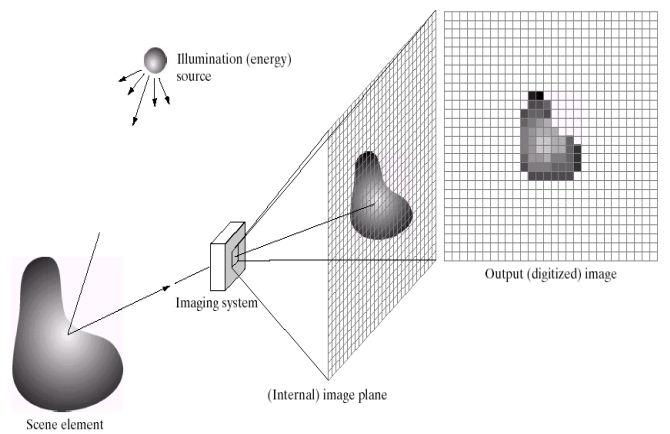

Fig:1 Digitized image

Image enhancement approaches fall into two broad categories: spatial domain methods and frequency domain methods. The term spatial domain refers to the image plane itself, and approaches in this category are based on direct manipulation of pixels in an image. Frequency domain processing techniques are based on modifying the Fourier transform of an image. Enhancement techniques based on various combinations of methods from these two categories are not unusual. There is no general theory of image enhancement. When an image is processed for visual interpretation, the viewer is the ultimate judge of how well a particular method works. One goal in image restoration is to remove the noise from the image in such a way that the "original" image is discernible.

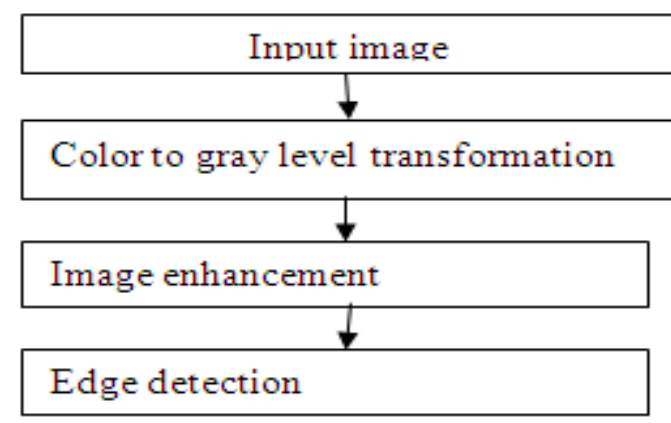

Fig:1 Proposed work 
Of course, "noise" is in the eye of the beholder, removing the "noise" is an image is important one.

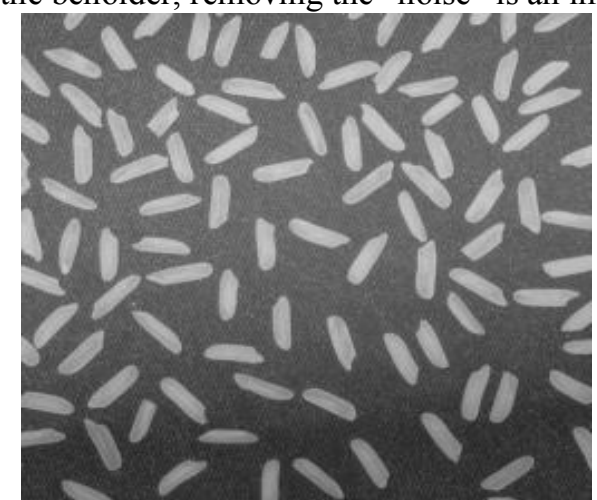

Fig:2(a) Original Image

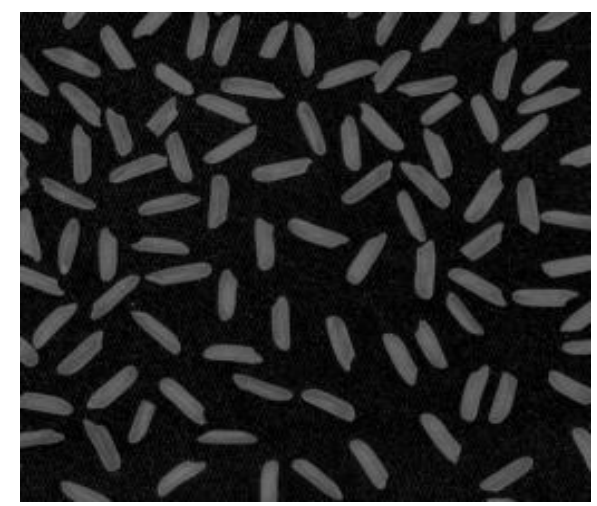

Fig:2(b) Enhanced image

Morphology is the study of the shape and form of objects. Morphological image analysis can be used to perform the operations like,

i. Object extraction

ii. Image enhancement operations, such as removal of small objects or noise from an image.

iii. Edge detectors, shape description.

In this Morphological processing first we "Read Image" from data base or appropriate file. Then we perform morphological opening operation to estimate the background illumination.

Morphological opening is erosion followed by dilation, using the same structuring element for both operations. The opening operation has the effect of removing objects that cannot completely contain the structuring element. Use the surf command to create a surface display of the background. The surf command creates colored parametric surfaces that enable you to view mathematical functions over a rectangular region. However, the surf function requires data of class double, so you first need to convert background using the double command to create a more uniform background, subtract the background image, background, from the original image. After subtraction, the image has a uniform background but is now a bit too dark. Use imadjust to adjust the contrast of the image, imadjust increases the contrast of the image by saturating $1 \%$ of the data at both low and high intensities and by stretching the intensity values to a required dynamic range. Then we perform the edge detector algorithm for identifying the object.

\section{SHAPE DETECTION}

Shape is not only, nut most powerful descriptor of image content. Shape is probably the most important property that is perceived about objects. It allows predicting more facts about an object than other features, e.g. colouring. Thus, recognizing shape is crucial for object recognition. In some applications it may be the only feature present, e.g. logo recognition.

Shape is not only perceived by visual means it is tactical sensors can also provide shape information that are processed in a similar way. 


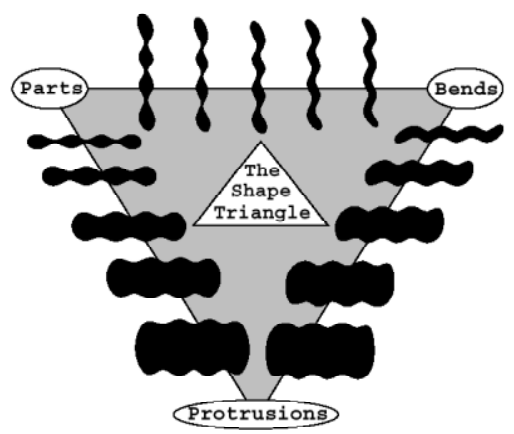

Fig:3 The shape Triangle

To detect the shape is the major problem. "Edge detection" is one of the techniques to identify the shape of the object. The points at which image brightness changes sharply are typically organized into a set of curved line segments termed edges. Edge detection is the fundamental tool on image processing to identify the object. Different types of edge detection techniques are available. Canny-edge detection and sobel-edge detection are most common edge detection techniques.

In Sobel edge detector, the task of edge detection is fulfilled by performing a $2 \mathrm{D}$ spatial gradient convolution operation on an image. This operator uses two convolution masks Gx and Gy. as shown fig.4

\begin{tabular}{|l|l|l|}
\hline-1 & 0 & 1 \\
\hline-2 & 0 & 2 \\
\hline-1 & 0 & 1 \\
\hline
\end{tabular}

\begin{tabular}{|l|l|l|}
\hline-1 & -2 & -1 \\
\hline 0 & 0 & 0 \\
\hline 1 & 2 & 1 \\
\hline
\end{tabular}

Fig:4 convolution masks (Gx and Gy) for sobel edge detector

Here, Gx and Gy are computed as,

$\mathrm{Gx}=-1 \mathrm{z} 1+1 \mathrm{z} 3-2 \mathrm{z} 4+2 \mathrm{z6}-1 \mathrm{z} 7+1 \mathrm{z} 9$
$\mathrm{~Gy}=1 \mathrm{z} 1+2 \mathrm{z} 2+1 \mathrm{z} 3-1 \mathrm{z} 7-2 \mathrm{z} 8-1 \mathrm{z} 9$

Where $\mathrm{Zi}, \mathrm{i}=1,2 \ldots 9$ are intensity levels of each pixel in the convolution window shown in fig4.1

\begin{tabular}{|l|l|l|}
\hline $\mathrm{Z} 1$ & $\mathrm{Z} 2$ & $\mathrm{Z} 3$ \\
\hline $\mathrm{Z4}$ & $\mathrm{Z} 5$ & $\mathrm{Z6}$ \\
\hline $\mathrm{Z7}$ & $\mathrm{Z} 8$ & $\mathrm{Z} 9$ \\
\hline
\end{tabular}

Sobel operator is very simple and effective way for finding the edges in image. The sobel operator is used mostly for detecting horizontal and vertical edges.

The Canny edge detector was considered to be an optimal edge detector, which satisfies all of the three performance criteria. The steps for algorithm are as follows:

1. The first step in this detector is to filter out noise in the image by using a Gaussian smoothing filter.

2. The second step in this edge detector is to locate the edge strength in the smoothed image by computing the image gradient, which helps to indicate where the actual edge is located.

3. The last step is to thin down the edges by tracking along the edge in the edge direction and set any pixel that is not at the maximum to be 0 , which is called non-maximum suppression. And finally edges are detected and link using pixel connectivity and double threshold that is, if the magnitude is above the high threshold, it is considered as an edge. If the magnitude is below the low threshold, it is considered as a non-edge.

This edge detector has the advantage that maximum edges get detected by using this edge detector. 


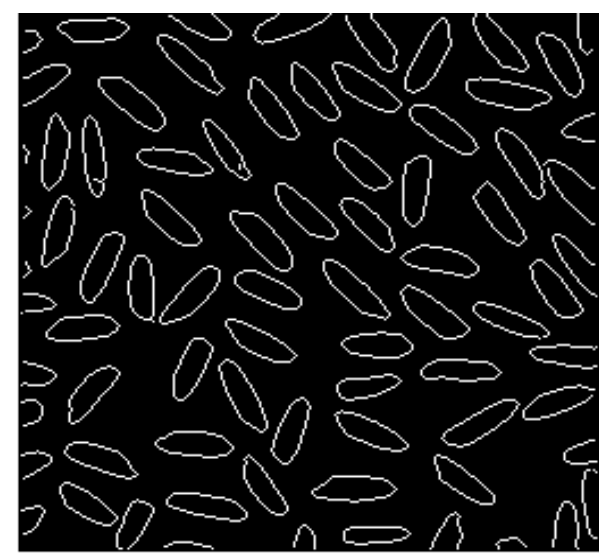

Fig: 5 Canny edge detection

To compare canny and sobel edge detection algorithm the canny edge detector is able to detect maximum number of edges. Canny's edge detector gives very good results for detecting horizontal and vertical edges .And at the same time it can detect the circular edges and edges at the corner.

In proposed work, the canny edge detection technique is used to detect the object.

\section{CONCLUSION}

MATLAB Software system developed with identifies the rice grains. Here, it displays the Output of the canny edge detector. It identifies the object in digital images. To identify the size of the each object also an important factor to finalize the object. So, Object counting and determine the breath and width of the object is extended work of this paper.

\section{REFERENCES}

[1] G.Ajay, M.Suneel, K.Kiran Kumar, P.Siva Prasad, Quality Evaluation of Rice Grains Using Morphological Methods, IJSCE, V-2, 2013.

[2] Jayme Garcia Arnal Barbedo, Digital image processing techniques for detecting, quantifying and classifying plant diseases. Journal on Springerplus V-2:660; 2013.

[3] R. Hussin, Digital Image Processing Techniques for Object Detection From Complex Background Image. Journal on Science Direct 2012.

[4] John Schmeelk, Edge Detectors on image processing 2011.

[5] Ali Javed, Khuram Ashfaq Qazi, Efficient Algorithm for Railway Track Detection, I.J Image, Graphics and Signal processing, 2012. 